\title{
Intravenous stroke thrombolysis after reversal of dabigatran effect by idarucizumab: first reported case in Hong Kong
}

WT Lo ${ }^{1}$, MRCP, FHKAM (Medicine), KF Ng², MRCS (Edin), FHKAM (Emergency Medicine), Simon CH Chan ${ }^{1}$, MRCP,

Vivian WY Kwok', MSc, CS Fong', MSc, ST Chan', MSc, Gordon CK Wong'리 MRCP, FHKAM (Emergency Medicine), WC Fong ${ }^{1}$, FRCP, FHKAM (Medicine)

Departments of ${ }^{1}$ Medicine and ${ }^{2}$ Accident and Emergency, Queen Elizabeth Hospital, Jordan, Hong Kong

*Corresponding author: fwcz01@ha.org.hk

Hong Kong Med J 2018;24:81-3

DOl: $10.12809 / \mathrm{hkmj} 166231$

\section{Case presentation}

A 78-year-old woman was diagnosed with atrial fibrillation in September 2015. An echocardiogram showed no evidence of valvular heart disease. She was prescribed dabigatran $110 \mathrm{mg}$ twice a day. In December 2016, she was admitted to our hospital for acute ischaemic stroke, presenting with a sudden onset of decreased level of consciousness. Apart from atrial fibrillation, she also had hypertension, diabetes mellitus, hyperlipidaemia, and a history of hip fracture with bilateral hip implants, requiring a rollator for walking.

On the day of admission, she was reported by her carer to be poorly responsive, with no verbal response and no spontaneous limb movement. She was last seen well 30 minutes previously. Physical examination revealed bilateral pinpoint pupils and no verbal response. She had slight flexion of her four limbs to pain and her National Institutes of Health Stroke Scale (NIHSS) score was 34. The blood pressure was $142 / 64 \mathrm{~mm} \mathrm{Hg}$. There was no hypoglycaemia. Naloxone was given with no improvement. Cerebral computed tomography (CT) showed no early infarct changes but bilateral subcortical white matter hypodensities, compatible with small vessel disease (Fig a). A clinical diagnosis was made of acute ischaemic brainstem stroke. The family confirmed that the patient had been taking dabigatran regularly as prescribed, with the last dose taken about 2 hours before symptom onset. She had taken no sedative medications.

After obtaining informed consent, an

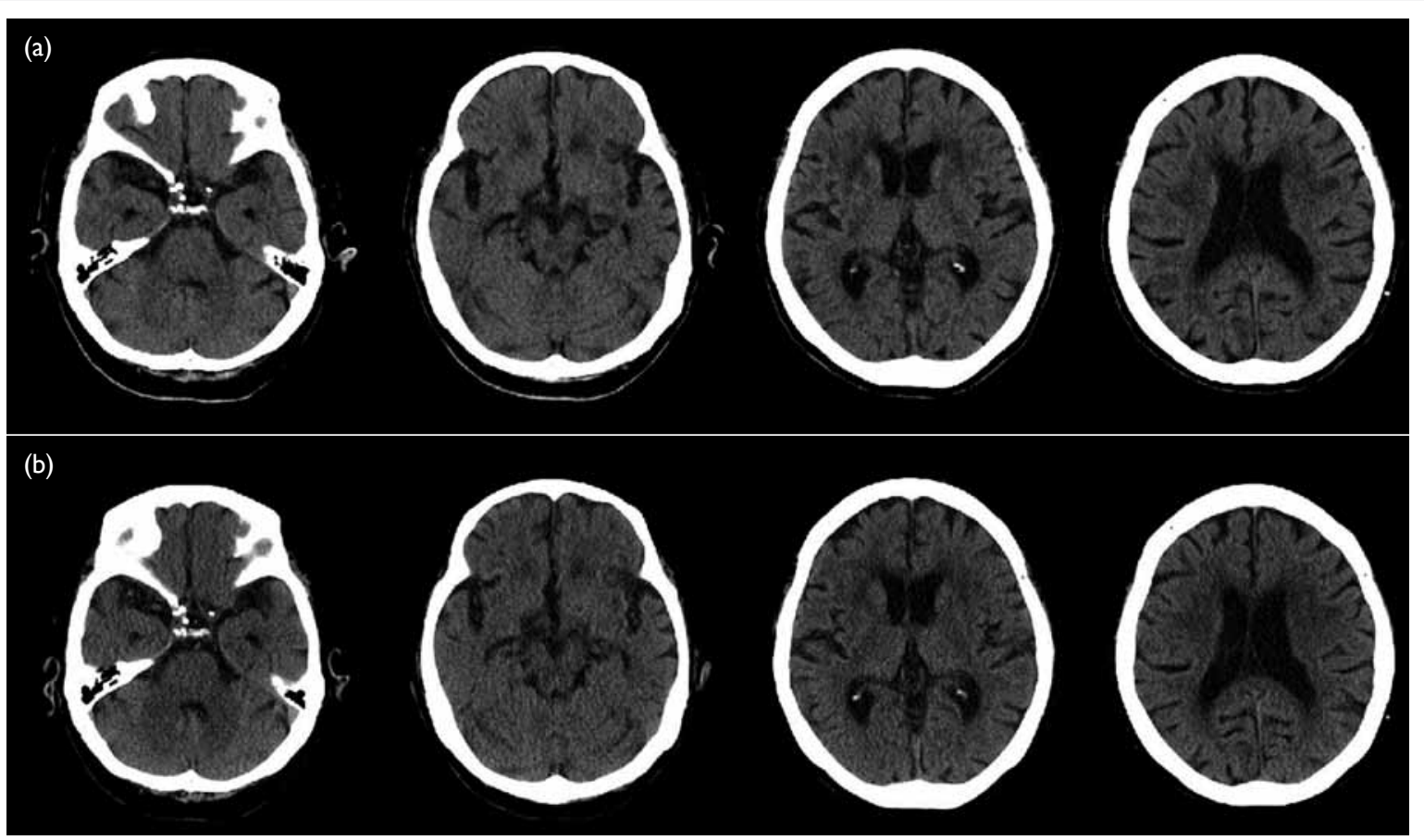

FIG. Computed tomography of the brain (a) pre-thrombolysis and (b) performed on day I post-thrombolysis 
intravenous bolus of $5 \mathrm{~g}$ idarucizumab was given. Blood for clotting profile, including thrombin time, was taken before treatment and 5 minutes afterwards. Intravenous recombinant tissue plasminogen activator ( $\mathrm{r}$-tPA) was then given at $0.6 \mathrm{mg} / \mathrm{kg}$ body weight 10 minutes after the start of idarucizumab injection, 2 hours from symptom onset. The prolonged activated partial thromboplastin time (APTT) and thrombin time (TT) normalised after administration of idarucizumab. The Table shows the clotting variables before and after idarucizumab. By the next morning, the patient had regained her speech although her response was slow with dysarthria and dysphagia. She could raise her four limbs against gravity, power being grade 3 . Followup CT at 24 hours post r-tPA showed no significant infarct and no bleeding (Fig b). Transcranial Doppler ultrasonography showed no evidence of significant vertebrobasilar occlusive disease. By day 2 , she had recovered further neurologically, with improvement in alertness, dysarthria and dysphagia, and could tolerate oral feeding. Limb power was grade 4+ over four limbs. Pupils were no longer pinpoint. She was recommenced on dabigatran $150 \mathrm{mg}$ twice a day on day 2 based on her age and creatinine clearance. The patient was discharged on day 7 with her neurological function returned to baseline.

\section{Discussion}

This is the first report in Hong Kong of the successful use of idarucizumab to reverse the anticoagulant effect of dabigatran, followed by intravenous thrombolysis with r-tPA for the treatment of ischaemic stroke. Novel oral anticoagulants (NOACs) are now increasingly used for the prevention of cardioembolic stroke in patients with non-valvular atrial fibrillation. Dabigatran, which acts as a thrombin inhibitor, is one such NOAC. Idarucizumab is a monoclonal antibody that has a high affinity for binding to the immunoglobulin $G$ fragment of dabigatran. A 5-g dose was shown to be able to reverse the anticoagulant effect of dabigatran within minutes and had a good safety profile in the study of Reversal Effects of Idarucizumab on Active Dabigatran (RE-VERSE AD trial). ${ }^{1}$

Patients who develop an acute ischaemic stroke while taking dabigatran are often excluded from treatment with intravenous thrombolysis owing to their bleeding risk. For patients who are not candidates for mechanical thrombectomy or who are in institutions where an endovascular thrombectomy service is not routinely available, giving intravenous thrombolysis after reversal of the anticoagulant effect is a treatment option. There is no pro-anticoagulant effect from idarucizumab itself, and an in-vitro study demonstrated no interaction between idarucizumab and r-tPA-induced thrombolysis. ${ }^{2}$ As far as we know, there have been five case reports at the time of writing of this article, all from German centres, that have reported the successful use of idarucizumab for this indication; none had any haemorrhagic complications., ${ }^{2,3}$ Four of these studies reported successful thrombolysis with good neurological recovery, whereas one study reported failed clinical improvement in a patient with infarcts in multiple territories. ${ }^{3}$ Diener et $\mathrm{al}^{4}$ have published an expert opinion on the management of these ischaemic strokes based on their experience in Germany. They proposed that for patients who have taken dabigatran in the preceding 24 hours from the time of assessment (or 96 hours if the creatinine clearance is $<30 \mathrm{~mL} / \mathrm{min}$ ), or for patients in whom time of last dabigatran dose is unknown and who have a prolonged APTT or TT, idarucizumab should be given if the patient is not a candidate for mechanical thrombectomy. In our institution, we do not have point-of-care devices to test clotting function, the turnaround time for APTT and TT results may be hours, and dabigatran concentrations cannot be measured in our laboratory. We therefore propose that for patients in whom time of last dabigatran dose is unknown, idarucizumab can still be considered with the family's consent to enable early intravenous thrombolysis. For the same reason, we did not wait for the results of APTT or TT to confirm reversal of dabigatran's effect before initiating intravenous thrombolysis. Idarucizumab rapidly and completely reversed the anticoagulant activity of dabigatran in $88 \%$ to $98 \%$ of patients in the RE-VERSE AD trial. ${ }^{1}$

Safety (avoidance of symptomatic intracranial haemorrhages) was our top concern for this patient. As she had multiple risk factors for intracranial haemorrhage (old age, high NIHSS score, cerebral white matter disease and on anticoagulant therapy), we gave r-tPA at a dose of $0.6 \mathrm{mg} / \mathrm{kg}$ body weight. This dose was associated with significantly fewer symptomatic intracranial haemorrhages in the

TABLE. Clotting variables before and after idarucizumab injection

\begin{tabular}{lccc}
\hline & Before idarucizumab & After idarucizumab & Reference range \\
\hline Activated partial thromboplastin time (s) & 50.7 & 29.5 & $25.8-33.8$ \\
Thrombin time (s) & 101.0 & 17.4 & $14.0-21.0$ \\
Prothrombin time (s) & 16.8 & 12.8 & $10.7-13.1$ \\
\hline
\end{tabular}


recent ENCHANTED trial. ${ }^{5}$

Following reversal of effect, dabigatran can be resumed as early as 24 hours afterwards. Although our patient had improved by the next day, she still had significant dysphagia and could not tolerate oral feeding. By the second day, she had improved further so dabigatran was resumed at a higher dose based on her age and renal function.

In conclusion, idarucizumab can be considered to reverse the anticoagulant effect of dabigatran in patients with ischaemic stroke within a therapeutic window, so that they may benefit from intravenous thrombolytic therapy.

\section{Declaration}

All authors have disclosed no conflicts of interest.

\section{References}

1. Pollack CV Jr, Reilly PA, Eikelboom J, et al. Idarucizumab for dabigatran reversal. N Engl J Med 2015;373:511-20.

2. Berrouschot J, Stoll A, Hogh T, Eschenfelder CC. Intravenous thrombolysis with recombinant tissuetype plasminogen activator in a stroke patient receiving dabigatran anticoagulant after antagonization with idarucizumab. Stroke 2016;47:1936-8.

3. Kafke W, Kraft P. Intravenous thrombolysis after reversal of dabigatran by idarucizumab: a case report. Case Rep Neurol 2016;8:140-4.

4. Diener HC, Bernstein R, Butcher K, et al. Thrombolysis and thrombectomy in patients treated with dabigatran with acute ischemic stroke: Expert opinion. Int J Stroke 2017;12:9-12.

5. Anderson CS, Robinson T, Lindley RI, et al. Low-dose versus standard-dose intravenous alteplase in acute ischemic stroke. N Engl J Med 2016;374:2313-23. 